\title{
Reconstruction of Conductivity Changes and Electrode Movements Based on EIT Temporal Sequences
}

\author{
Tao Dai, Camille Gómez-Laberge and Andy Adler \\ Systems and Computer Engineering, Carleton University, Ottawa, Canada \\ E-mail: adler@sce.carleton.ca
}

\begin{abstract}
Electrical impedance tomography (EIT) reconstructs a conductivity change image within a body from electrical measurements on body surface; while it has relatively low spatial resolution, it has a high temporal resolution. One key difficulty with EIT measurements is due to the movement and position uncertainty of the electrodes, especially due to breathing and posture change. In this paper, we develop an approach to reconstruct both the conductivity change image and the electrode movements from the temporal sequence of EIT measurements. Since both the conductivity change and electrode movement are slow with respect to the data frame rate, there are significant temporal correlations which we formulate as priors for the regularized image reconstruction model. Image reconstruction is posed in terms of a regularization matrix and Jacobian matrix which are augmented for the conductivity change and electrode movement, and then further augmented to concatenate the $d$ previous and future frames. Results are shown for simulation, phantom and human data, and show that the proposed algorithm yields improved resolution and noise performance in comparison to a conventional one-step reconstruction method.
\end{abstract}

Keywords: Electrical Impedance Tomography, regularization, image reconstruction, electrode movement, temporal correlation.

\section{Introduction}

Electrical Impedance Tomography (EIT) calculates an estimate of the conductivity distribution within a body based on current stimulations and voltage measurements on the body surface. EIT imaging has low spatial resolution, however, EIT can have excellent temporal resolution. Some recent systems have frame rates up to 1000 fps (Wilkinson et al 2005). Such high temporal resolution makes EIT a promising technology to monitor fast physiological events which affect the conductivity distribution.

For cardiac activities, EIT can locally determine impedance variations in ventricular or atrial regions during the cardiac cycle (Eyuboglu et al 1989) and calculate cardiac parameters such as stroke volume (Vonk-Noordegraaf et al 2000). For pulmonary function monitoring, EIT has been demonstrated as an effective tool due to the 
conductivity variation of lungs is highly related to the air ventilation (Dijkstra et al 1993), blood infusion (McArdle et al 1988), and intrathoracic fluid volumes (Campbell et al 1994). EIT is helpful for imaging rapid conductivity changes due to brain neuronal activity, which occur within a timescale of milliseconds, such as acute blood infusion/exfusion from visually evoked responses (Holder et al 1987 and Tidswell et al 2001).

Due to the diffusive propagation of electrical current in the human body, EIT is a soft field tomography modality. Compared with the number of pixels/voxels to be reconstructed, the amount of electrodes that can be applied on body surface is relatively small. Thus, the reconstruction of an unknown internal conductivity distribution from boundary data is severely ill-conditioned (Lionheart et al 2005). In order to calculate a "reasonable" image, regularization techniques are required. Such regularized image reconstructions can be statistically formulated in terms of a priori information about image element values and the correlations among them. In many EIT algorithms, the zeroth order Tikhonov (Vauhkonen et al 1998a), discrete Laplacian filter (Polydorides and Lionheart et al 2002) and the NOSER priors (Cheney et al 1990, Graham and Adler 2007) are commonly used. Those regularization priors treat all images independently from each other. However, it is clear that images within a certain temporal distance are not independent but do contain useful temporal correlations, especially for high speed EIT. This type of temporal correlation was exploited by Adler et al (2007) to improve EIT image noise performance and resolution.

The position uncertainty of electrodes is a principle source of artefacts and reconstruction errors. Difference EIT is less sensitive to electrode position uncertainty assuming the electrodes do not move during measurement (Barber and Brown 1988). However, this assumption is not valid in medical applications, such as cardiopulmonary imaging, in which the chest moves during breathing and/or posture change. Harris et al (1988), Adler et al (1996) and Zhang and Patterson (2005) showed the electrode movement introduced by thoracic variation had a significant effect on EIT imaging.

In this paper, we address two issues in EIT, the electrode position uncertainty and data noise, by taking advantage of the high frame rate of modern EIT systems. Previously, we developed an approach to reconstruct conductivity change and electrode movement from a single frame of EIT data using an augmented Jacobian and prior matrix (Soleimani et al 2006). One limitation of this approach is that reconstructed electrode movements can often be noisy. This paper develops an algorithm to improve reconstructions of both electrode movements and conductivity changes by considering correlations between reconstructions in successive data frames. We take advantage of the fact that with fast EIT systems, the boundary shape and internal conductivities change relatively slowly, and may be formulated as a priori constraints in the image reconstruction model. This paper proposes to reconstruct EIT images via an inverse problem with a regularization prior that accounts for both spatial and temporal correlations among image elements and electrode movements. This algorithm is verified by numerical simulation, saline phantom data and in vivo human measurement. 


\section{Methods}

An EIT system has $n_{E}$ electrodes applied on a body surface in a plane using the adjacent current stimulation and voltage measurement. Also $n_{E}$ current stimulation patterns are sequentially applied and each of them takes $n_{E}-3$ differential measurements. Each data frame measures a vector, $\mathbf{v} \in \mathbb{R}^{n_{M}}$, of $n_{M}=n_{E}\left(n_{E}-3\right)$ data points (some of which are redundant if the medium is not changing). Difference EIT calculates difference data $\mathbf{y}$, $\left(\mathbf{y}_{i}=\mathbf{v}_{i}-\mathbf{v}_{0}\right.$, where $i$ is the time index. To improve its precision, the reference signal $\mathbf{v}_{0}$ is typically the average over many data frames that are acquired when the measured object may be assumed to be stable. Therefore, $\mathbf{v}_{0}$ is assumed noise-free.

The body investigated is modelled using a finite element model (FEM) that discretizes the body conductivity into $n_{N}$ piecewise smooth elements, represented by a vector $\boldsymbol{\sigma} \in \mathbb{R}^{n_{N}}$. Difference EIT calculates a vector of conductivity changes, $\mathbf{x}_{i}=\boldsymbol{\sigma}_{i}-\boldsymbol{\sigma}_{0}$ between the present conductivity distribution, $\boldsymbol{\sigma}_{i}$, and that at the reference measurement $\boldsymbol{\sigma}_{0}$. For small variations around the reference conductivity $\boldsymbol{\sigma}_{0}$, the relationship between $\mathbf{x}$ and $\mathbf{y}$ can be linearized so that the difference EIT forward model is:

$$
\mathbf{y}=\mathbf{J x}+\mathbf{n}
$$

where $\mathbf{J} \in \mathbb{R}^{n_{M} \times n_{N}}$ is the Jacobian or sensitivity matrix and $\mathbf{n} \in \mathbb{R}^{n_{M}}$ is the vector of measurement noise, which is assumed to be uncorrelated white Gaussian. The Jacobian is calculated from the FEM as $\mathbf{J}_{i j}=\left.\frac{\partial \mathbf{y}_{i}}{\partial \mathbf{x}_{j}}\right|_{\boldsymbol{\sigma}_{0}}$, and depends on the FEM, current injection patterns, the reference conductivity and the electrode models. This system is underdetermined since $n_{N}>n_{M}$. This problem is commonly solved using regularization techniques in order to calculate an estimated conductivity change $\hat{\mathbf{x}}$, which is both faithful to the measurements $\mathbf{y}$ and to a priori constraints on a "reasonable" image.

Under the condition that the system is relatively stable ( $\mathbf{J}$ is constant), a sequence of difference data $\mathbf{y}_{i}$ are obtained. As long as the conductivity of the body measured doesn't change too rapidly, it is reasonable to expect that a certain number $d$ of adjacent frames in the past and from the future provide useful hints about the current image. Labelling the current instant as $t$, we then seek to estimate $\hat{\mathbf{x}}_{t}$ from the data $\left[\mathbf{y}_{t-d} ; \ldots ; \mathbf{y}_{t-1} ; \mathbf{y}_{t} ; \mathbf{y}_{t+1} ; \ldots ; \mathbf{y}_{t+d}\right]$.

In the subsequent sections we consider the following methods: 2.1) Traditional GN inverse, using $\mathbf{y}_{t}$ only, to reconstruct conductivity change. 2.2) GN inverse calculating both conductivity change and electrode movement, using $\mathbf{y}_{t}$ only, to reconstruct both conductivity change and electrode movement. 2.3) Temporal GN inverse, using $\mathbf{y}_{t-d} \cdots \mathbf{y}_{t+d}$, to reconstruct conductivity change only based on a temporal prior model. 2.4) Temporal inverse on both conductivity change and electrode movement, using $\mathbf{y}_{t-d} \cdots \mathbf{y}_{t+d}$, to reconstruct both conductivity change and electrode movement based on a temporal prior model. 


\subsection{One-step linear Gauss-Newton (GN) solver}

The regularized image reconstruction based on the one-step linearized GN method was first introduced into EIT by Yorkey et al (1987) and has been widely used (e.g., Cheney et al 1990; Adler and Guardo, 1996). It addresses the inverse solution as a linear reconstruction matrix and allows use of advanced regularization methods to solve the inverse problem. By using a precalculated reconstruction matrix, it can realize rapid, real-time imaging. The GN inverse problem estimates a solution $\hat{\mathbf{x}}$ by minimizing

$$
\|\mathbf{y}-\mathbf{J} \hat{\mathbf{x}}\|_{\Sigma_{n}^{-1}}^{2}+\left\|\mathbf{x}-\mathbf{x}^{\circ}\right\|_{\Sigma_{x}^{-1}}^{2}
$$

where $\Sigma_{n} \in \mathbb{R}^{n_{M} \times n_{M}}$ is the covariance matrix of the measurement noise $\mathbf{n}$. Since noise channels are independent, $\Sigma_{n}$ is a diagonal matrix with $\left[\Sigma_{n}\right]_{i, i}=\sigma_{i}^{2}$, where $\sigma_{i}^{2}$ is the noise variance at channel $i$. Here $\Sigma_{x} \in \mathbb{R}^{n_{N} \times n_{N}}$ is the covariance matrix of the desired image and $\mathbf{x}^{\circ}$ represents the expected value of image, which is zero for difference EIT.

Instead of calculating $\Sigma_{n}$ and $\Sigma_{x}$, we heuristically model them from a priori considerations by introducing $\mathbf{W}=\sigma_{n}^{2} \Sigma_{n}^{-1}$ and $\mathbf{R}=\sigma_{x}^{2} \Sigma_{x}^{-1}$. Here $\sigma_{n}$ is the average measurement noise amplitude and $\sigma_{x}$ is the a priori amplitude of conductivity changes. The measurement accuracy is modelled by $\mathbf{W}$. For uncorrelated noise, each diagonal element of $\mathbf{W}$ is proportional to the corresponding channel signal-to-noise-ratio (SNR). For difference EIT with identical channels, $\mathbf{W}$ is an identity matrix; or else, it may be measured during the system calibration test (the identity matrix is used in this paper for simplicity). The regularization matrix $\mathbf{R}$ may be understood to statistically model the amplitudes and interactions of image elements. Simply, $\mathbf{R}$ may consider all elements equally alike and independent (or only locally dependent). More sophisticated models (e.g., Dai et al 2007) may consider smooth distributions more likely than rapidly changing ones and using temporal/spatial correlations.

From (2), a linearized one-step inverse solution is obtained as

$$
\hat{\mathbf{x}}=\left(\mathbf{J}^{T} \mathbf{W} \mathbf{J}+\lambda^{2} \mathbf{R}\right)^{-1} \mathbf{J}^{T} \mathbf{W} \mathbf{y}=\mathbf{B} \mathbf{y}
$$

where $\lambda=\sigma_{n} / \sigma_{x}$ is the regularization parameter, or hyperparameter, which controls the trade-off between resolution and noise attenuation in the reconstructed image. Here $\mathbf{B}=\left(\mathbf{J}^{T} \mathbf{W} \mathbf{J}+\lambda^{2} \mathbf{R}\right)^{-1} \mathbf{J}^{T} \mathbf{W}$ is the linear, one-step reconstruction matrix.

Assuming that image elements are independent and have an identical expected magnitude, $\mathbf{R}$ becomes an identity matrix I and (3) uses zeroth order Tikhonov regularization. For EIT, such solutions tend to push reconstructed noise toward the boundary, since the measured data are much more sensitive to boundary elements than deep elements. In order to compensate the sensitivity discrepancy, $\mathbf{R}$ may be scaled by the sensitivity of elements, so that $\mathbf{R}$ is a diagonal matrix with elements $[\mathbf{R}]_{i, i}=\left[\mathbf{J}^{T} \mathbf{J}\right]_{i, i}^{p}$. This is the NOSER prior (Cheney et al 1990) with an exponent $p$. In this paper, the NOSER prior is used in all tested algorithms. The exponent is chosen as $p=0.5$ heuristically, as a compromise between the pushing noise to the boundary $(p=0)$ or to the centre $(p=1)$. 
The term inverted in (3) is of size $n_{N} \times n_{N}$. The matrix $\mathbf{B}$ can be rewritten using the data form (Adler et al 2007:

$$
\mathbf{B}=\mathbf{P J}^{T}\left(\mathbf{J P J} \mathbf{J}^{T}+\lambda^{2} \mathbf{V},\right)^{-1}
$$

where $\mathbf{P}=\mathbf{R}^{-1}=\Sigma_{x} / \sigma_{x}^{2}$ and $\mathbf{V}=\mathbf{W}^{-1}=\Sigma_{n} / \sigma_{n}^{2}$. In (4), the size of the inverted matrix is reduced to $n_{M} \times n_{M}$. This is important for large scale models, such as 3D EIT models and the temporal inverse, which is introduced below.

\subsection{Reconstruction of conductivity change and electrode movement}

One of the primary difficulties interpreting EIT images in clinics is the movement of electrodes from breathing or posture changes. The inaccurately modelled electrode placement introduces severe artefacts in reconstructed images. In order to solve this modelling error, Soleimani et al (2006) developed an algorithm to reconstruct both the conductivity change and the electrode movement simultaneously by combining conductivity changes and electrode movement reconstructions into a single inversion process. An electrode displacement vector $\Delta \mathbf{r} \in \mathbb{R}^{n_{D} n_{E}}$, where $n_{D}$ is the model dimension ( 2 or 3 for $2 \mathrm{D}$ or $3 \mathrm{D}$, respectively), is concatenated to the difference conductivity vector $\mathbf{x}_{c}$. Thus the augmented vector to be reconstructed is $\mathbf{x}=$ $\left[\begin{array}{ll}\mathbf{x}_{c}^{T} & \Delta \mathbf{r}^{T}\end{array}\right]^{T} \in \mathbb{R}^{n_{N}+n_{D} n_{E}}$. The augmented Jacobian becomes $\mathbf{J}=\left[\mathbf{J}_{c} \mathbf{J}_{m}\right] \in$ $\mathbb{R}^{n_{M} \times\left(n_{N}+n_{D} n_{E}\right)}$, where $\mathbf{J}_{c} \in \mathbb{R}^{n_{M} \times n_{N}}$ and $\mathbf{J}_{m} \in \mathbb{R}^{n_{M} \times n_{D} n_{E}}$ are the conductivity and electrode movement Jacobians, respectively. The overall prior matrix is $\mathbf{R}=$ $\operatorname{diag}\left(\mathbf{R}_{c}, \mathbf{R}_{m}\right) \in \mathbb{R}^{\left(n_{N}+n_{D} n_{E}\right) \times\left(n_{N}+n_{D} n_{E}\right)}$, where $\mathbf{R}_{c} \in \mathbb{R}^{n_{N} \times n_{N}}$ and $\mathbf{R}_{m} \in \mathbb{R}^{n_{D} n_{E} \times n_{D} n_{E}}$ are conductivity and electrode movement prior matrices, respectively. Finally, the onestep reconstruction is the same as (3) with rebuilt $\mathbf{J}, \mathbf{x}$ and $\mathbf{R}$.

The electrode movement Jacobian $\left(\mathbf{J}_{m}\right)$ is calculated using the rank one perturbation technique (Olsen and Gopinath 2004), as implemented by Gómez-Laberge and Adler (2007).

The prior matrix statistically describes the "desired" values of conductivity changes and electrode movements. The upper $n_{N} \times n_{N}$ part of $\Sigma_{x}^{-1}$ in (2) represents covariance of finite element conductivity changes, while the lower $n_{D} n_{E} \times n_{D} n_{E}$ part represents the covariance of electrode movements. By carefully establishing prior matrices for specific applications, different reconstruction performances can be obtained (Adler and Lionheart 2006). With a similar purpose to that described in Section 2.1, concerning the sensitivity discrepancy, we need compensations on "overweighted" elements which have high sensitivities. The NOSER prior is used in this paper. Suppose the calculated augmented Jacobian is $\mathbf{J}=\left[\mathbf{J}_{c} \mathbf{J}_{m}\right]$, then the $\mathbf{R}$ is built so that $[\mathbf{R}]_{i, i}=\left[\mathbf{J}^{T} \mathbf{J}\right]_{i, i}^{1 / 2}$.

\subsection{Temporal one-step solver}

Adler et al (2007) proposed a temporal image reconstruction algorithm that calculates the image at a current frame considering data from adjacent frames. This approach differs from the Kalman filter based algorithms (Vauhkonen et al 1998b), which estimate 
image $\mathbf{x}_{t}$ based on measurements $\mathbf{y}_{t}$ and the previous image estimate $\mathbf{x}_{t-1}$. The temporal solver treats the estimate of the image frame sequence as a single inverse problem with a regularization prior that accounts for both spatial and temporal correlations between image elements.

2.3.1. Temporal reconstruction. The temporal solver considers a sequence of $2 d+1$ data frames from $t-d$ to $t+d$ around the current frame $t$. Given a vertically concatenated data frame sequence $\tilde{\mathbf{y}}_{t}=\left[\begin{array}{llllll}\mathbf{y}_{t-d}^{T} & \ldots & \mathbf{y}_{t}^{T} & \ldots & \mathbf{y}_{t+d}^{T}\end{array}\right]^{T}$ and the corresponding concatenated image sequence $\tilde{\mathbf{x}}_{t}=\left[\begin{array}{llllll}\mathbf{x}_{t-d}^{T} & \cdots & \mathbf{x}_{t}^{T} & \ldots & \mathbf{x}_{t+d}^{T}\end{array}\right]^{T}$, the direct temporal forward model is rewritten from $(1)$ as

$$
\tilde{\mathbf{y}}_{t}=\tilde{\mathbf{J}} \tilde{\mathbf{x}}_{t}+\tilde{\mathbf{n}},
$$

where $\tilde{\mathbf{n}}=\left[\begin{array}{llllll}\mathbf{n}_{t-d}^{T} & \ldots & \mathbf{n}_{t}^{T} & \ldots & \mathbf{n}_{t+d}^{T}\end{array}\right]^{T}$. We consider that $\mathbf{J}$ is time invariant. Thus, $\tilde{\mathbf{J}}=\mathbf{I} \otimes \mathbf{J}$, where the identity matrix $\mathbf{I}$ is of size $2 d+1$, and $\otimes$ is the Kronecker product.

There exists an inter-frame correlation between two images temporally close to each other. As images become further separated in time, the correlation decreases; for a separation of $\delta$, the correlation is $\exp (-|\delta| / \gamma)$, where $\gamma$ is the temporal exponential decay factor in units of frames. Frames with a large time difference $|\delta|>d$, are considered independent. The one-step inverse (4) then becomes

$$
\tilde{\mathbf{B}}=\tilde{\mathbf{P}} \tilde{\mathbf{J}}^{T}\left(\tilde{\mathbf{J}} \tilde{\mathbf{P}} \tilde{\mathbf{J}}^{T}+\lambda^{2} \tilde{\mathbf{V}}\right)^{-1}
$$

where $\tilde{\mathbf{V}}=\mathbf{I} \otimes \mathbf{V}$. Also $\tilde{\mathbf{P}}=\boldsymbol{\Gamma} \otimes \mathbf{P}$, where $\boldsymbol{\Gamma}$ is the temporal weight matrix of an image sequence $\tilde{\mathbf{x}}$ and is defined to have the form

$$
[\boldsymbol{\Gamma}]_{i, j}=\exp \left(-\frac{|i-j|}{\gamma}\right) \quad i, j=-d, \ldots, d .
$$

From (6) and (7),

$$
\tilde{\mathbf{B}}=\left[\mathbf{\Gamma} \otimes\left(\mathbf{P} \mathbf{J}^{T}\right)\right]\left[\boldsymbol{\Gamma} \otimes\left(\mathbf{J P J}^{T}\right)+\lambda^{2}(\mathbf{I} \otimes \mathbf{V})\right]^{-1} .
$$

Given $\tilde{\mathbf{B}}$, the one-step solution $\hat{\mathbf{x}}_{t}$ for the current image is rewritten as

$$
\hat{\mathbf{x}}_{t}=\tilde{\mathbf{B}}_{0} \tilde{\mathbf{y}}_{t}
$$

where $\tilde{\mathbf{B}}_{0}$ occupies the rows $n_{M} \times d+1, \ldots, n_{M} \times(d+1)$ of $\tilde{\mathbf{B}}$.

2.3.2. Parameter selection. The $\gamma$ may be considered a hyperparameter of the system: it depends on the data acquisition frame rate, the speed of underlying conductivity changes and the system noise level. The estimation of $\gamma$ can be addressed as a method of "kernel smoothing" (Fahrmeir and Tutz, 1994). The object function to be minimized can be averaged squared error, mean average squared error, or average predictive squared error, etc. In this paper, we take covariance on both sides of (5). We have

$$
\Sigma_{\tilde{\mathbf{y}}}=\tilde{\mathbf{J}} \Sigma_{\tilde{\mathbf{x}}} \tilde{\mathbf{J}}^{t}+\Sigma_{\tilde{\mathbf{n}}}
$$


The optimal $\gamma$ is chosen so that the covariance error $\Sigma_{\tilde{\mathbf{y}}}-\left(\tilde{\mathbf{J}} \Sigma_{\tilde{\mathbf{X}}} \tilde{\mathbf{J}}^{t}+\Sigma_{\tilde{\mathbf{n}}}\right)$ is minimized as

$$
\gamma=\underset{\gamma}{\operatorname{argmin}}\left\|\Sigma_{\tilde{\mathbf{y}}}-\Sigma_{\tilde{\mathbf{n}}}-\tilde{\mathbf{J}} \Sigma_{\tilde{\mathbf{x}}} \tilde{\mathbf{J}}^{t}\right\|_{F}^{2}
$$

where the $\|\cdot\|_{F}$ is the Frobenius norm. Since $\Sigma_{\tilde{\mathbf{X}}}=\mathbf{\Gamma} \otimes \Sigma_{\mathbf{X}}$ and $\tilde{\mathbf{J}}=\mathbf{I} \otimes \mathbf{J}$, (11) becomes

$$
\gamma=\underset{\gamma}{\operatorname{argmin}}\left\|\Sigma_{\tilde{\mathbf{y}}}-\Sigma_{\tilde{\mathbf{n}}}-\mathbf{\Gamma} \otimes\left(\mathbf{J} \Sigma_{\mathbf{X}} \mathbf{J}^{t}\right)\right\|_{F}^{2}
$$

By taking covariance on both sides of (1), we have

$$
\Sigma_{\mathbf{y}}=\mathbf{J} \Sigma_{\mathbf{X}} \mathbf{J}^{t}+\Sigma_{\mathbf{n}}
$$

so that $\mathbf{J} \Sigma_{\mathbf{X}} \mathbf{J}^{t}=\Sigma_{\mathbf{y}}-\Sigma_{\mathbf{n}}$. We also have $\Sigma_{\tilde{\mathbf{n}}}=\mathbf{I} \otimes \Sigma_{\mathbf{n}}$ and $\Sigma_{\tilde{\mathbf{y}}}=\boldsymbol{\Gamma}_{\mathbf{y}} \otimes \Sigma_{\mathbf{y}}$, where $\boldsymbol{\Gamma}_{\mathbf{y}} \in \mathbb{R}^{(2 d+1) \times(2 d+1)}$ is the correlation matrix of $\tilde{\mathbf{y}}$. Thus the optimal $\gamma$ is calculated by

$$
\gamma=\underset{\gamma}{\operatorname{argmin}}\left\|\boldsymbol{\Gamma}_{\mathbf{y}} \otimes \Sigma_{\mathbf{y}}-\mathbf{I} \otimes \Sigma_{\mathbf{n}}-\boldsymbol{\Gamma} \otimes\left(\Sigma_{\mathbf{y}}-\Sigma_{\mathbf{n}}\right)\right\|_{F}^{2} .
$$

Here $\boldsymbol{\Gamma}_{\mathbf{y}}$ and $\Sigma_{\mathbf{y}}$ can be calculated directly from the data; $\Sigma_{\mathbf{n}}$ can be obtained in EIT system calibration phase. For computational efficiency, (14) can be rewritten as

$$
\gamma=\underset{\gamma}{\operatorname{argmin}}\left\|\boldsymbol{\Gamma}_{\mathbf{y}}\right\| \Sigma_{\mathbf{y}}\left\|_{F}^{2}-\mathbf{I}\right\| \Sigma_{\mathbf{n}}\left\|_{F}^{2}-\boldsymbol{\Gamma}\right\| \Sigma_{\mathbf{y}}-\Sigma_{\mathbf{n}}\left\|_{F}^{2}\right\|_{F}^{2},
$$

where $\boldsymbol{\Gamma}_{\mathbf{y}},\left\|\Sigma_{\mathbf{y}}\right\|_{F}^{2},\left\|\Sigma_{\mathbf{n}}\right\|_{F}^{2}$ and $\left\|\Sigma_{\mathbf{y}}-\Sigma_{\mathbf{n}}\right\|_{F}^{2}$ may be precalculated. Since $\boldsymbol{\Gamma}$ is relatively small (of size $(2 d+1) \times(2 d+1)$ ), this optimization is performed directly by the bisection search between limits.

\subsection{Temporal reconstruction of conductivity change and electrode movement}

The temporal solver for reconstruction of both conductivity change and electrode movement is formulated in terms of a regularized one-step inverse (8), in which the Jacobian $\mathbf{J}$ is rebuilt as $\left[\mathbf{J}_{c} \mathbf{J}_{m}\right]$ and

$$
\tilde{\mathbf{J}}=\mathbf{I} \otimes\left[\mathbf{J}_{c} \mathbf{J}_{m}\right] .
$$

The augmented prior matrix is computed as NOSER prior from the precalculated Jacobian and

$$
\tilde{\mathbf{P}}=\Gamma \otimes\left[\mathbf{J}^{T} \mathbf{J}\right]_{i, i}^{-1 / 2}
$$

The inverse is further processed in terms of an augmented image $\tilde{\mathbf{x}}$ and measurement vector $\tilde{\mathbf{y}}(9)$, which concatenate the values from $d$ previous and $d$ future frames.

\subsection{Method: experiments}

Data from numerical simulations, a saline phantom measurement and in vivo human measurement were used to test these algorithms proposed. 
2.5.1. Numerical simulation. Numerical simulation data were obtained from a 2D FEM model with 5184 elements, with homogeneous conductivity $\sigma_{h}=1$. Illustrated as Figure 1(a), A unit radius circular model with 16 electrodes was built and an adjacent stimulation and measurement pattern was applied. In this model a conductive $\left(1.2 \times \sigma_{h}\right)$ spherical object with 0.05 unit radius rotated clockwise along a trajectory that had a radius of $2 / 3$ unit, Two hundred frames were taken per movement cycle. A distortion (horizontal compression and vertical elongation) was applied with distortion amplitudes gradually increased from 0 at the first frame to $1 \%$ of the model diameter at the end $\left(200^{\text {th }}\right.$ frame).

2.5.2. Phantom measurement. The saline phantom is a plastic cylindrical tank with $30 \mathrm{~cm}$ diameter and $30 \mathrm{~cm}$ height, being filled with $0.9 \%$ saline solution to the $20 \mathrm{~cm}$ height. Sixteen stainless steel electrodes were equidistantly placed around the circumference at a vertical position of $10 \mathrm{~cm}$. EIT data were acquired using the Goe-MF II EIT system (Viasys Healthcare, Höchberg, Germany) using an adjacent stimulation and measurement pattern. First, data of a homogeneous background $\mathbf{y}_{h}$ were acquired and processed by ensemble averaging; then two small non-conductive spherical objects of $2 \mathrm{~cm}$ radius were statically suspended at the same level of the electrode plane at positions $(0,7 \mathrm{~cm})$ and $(7 \mathrm{~cm}, 0)$ as illustrated in Figure $1(\mathrm{~b})$. A compression was applied on the top of the phantom along the $x$-axis so that the cross section of the tank became an ellipse with a minor axis of $25 \mathrm{~cm}$. Since the electrode plane was at $1 / 3$ of the tank height, the maximum movement of electrodes was about $1.7 \mathrm{~cm}$. This phantom was gradually compressed during the first 6 seconds and held with elliptical shape afterwards.

2.5.3. In vivo human measurement. EIT data were acquired from a healthy adult using the Goe-MF II EIT system (Viasys Healthcare, Höchberg, Germany). Adjacent stimulation and measurement patterns were applied. The data acquisition speed was 13 fps. Sixteen $3 M^{T M}$ Red-Dot $\mathrm{Ag} / \mathrm{AgCl}$ electrodes were equidistantly attached around the thoracic circumference at a horizontal plane that was $1 \mathrm{~cm}$ under the nipple line. The $1^{\text {st }}$ Electrode was in the centre of the sternum. Other electrodes were subsequently placed towards the subject's right side so that the $5^{\text {th }}$ electrode was under the right armpit, the $9^{\text {th }}$ electrode on the spine and the $13^{\text {th }}$ electrode under the left armpit. A $17^{\text {th }}$ electrode was attached at lower right waist as reference/ground. All measurements were taken while the subject was standing and conducting deep breaths to total lung capacity.

\section{Results}

The forward and inverse calculations used the EIDORS software (Adler and Lionheart 2006). The numerical and phantom data were reconstructed on a circular 576 element model and the in vivo data was on a 2D thoracic 576 element model. For the numerical 


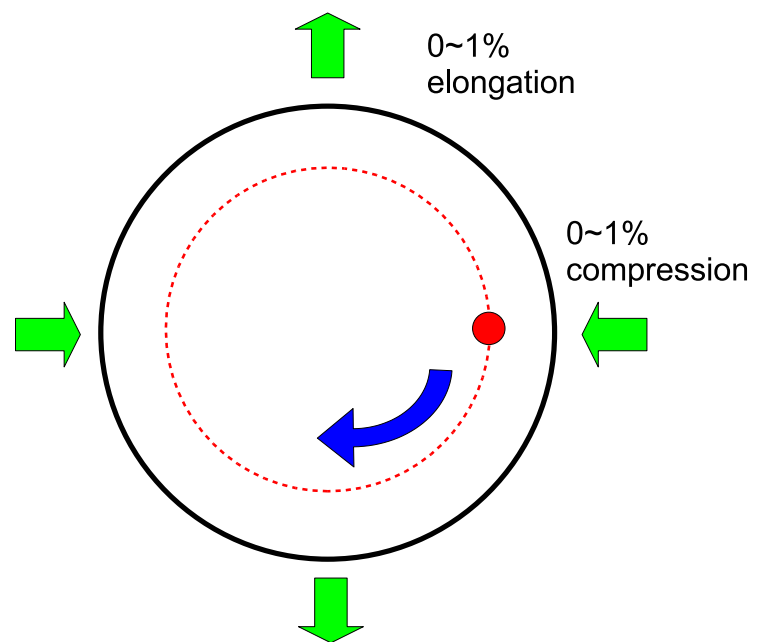

(a)

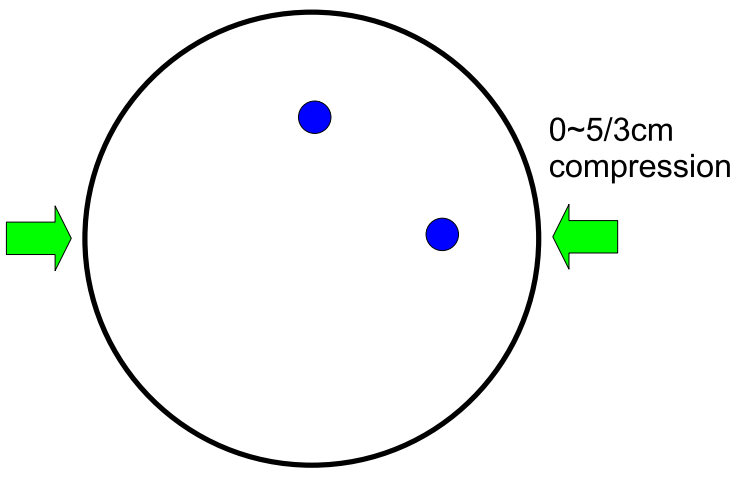

(b)

Figure 1. Illustration of 2D numerical model simulation and saline phantom measurement. Green arrows indicate directions of boundary distortion. (a) one conductive target rotates clockwise (trajectory as dashed circle). Distortion amplitude increases linearly from 0 to $1 \%$ of model diameter. Background conductivity $\sigma_{h}=1$ and the target conductivity is $1.2 \times \sigma_{h}$. The illustrated target position is the start/final position of one full cycle. (b) two non-conductive targets statically suspended in a saline phantom at the level of the electrode plane. The phantom diameter is $30 \mathrm{~cm}$, the maximum distortion of the boundary is $5 / 3 \mathrm{~cm}$.

simulation, the choice of 576 elements for the inverse mesh was to differ from the simulation model to avoid the inverse crime. Gaussian white noise was added to numerical simulated data with noise level $S N R$, defined as $\overline{\mathbf{y}} / \sigma_{\mathbf{n}}$, where $\sigma_{\mathbf{n}}$ was the standard deviation of the added noise and $\overline{\mathbf{y}}$ was the mean value of the difference signal. Different random seeds were tested and showed similar results. Four algorithms were evaluated with different regularization methods: 1) GN solver (Sec.2.1); 2) temporal solver (Sec.2.3); 3) electrode movement solver (Sec.2.2); and 4) temporal and electrode movement solver (Sec.2.4).

To better understand the effect of the model distortion on reconstructed image, we used the traditional GN solver to calculate images of a distorted homogeneous medium (Figure 2, left). The 2D model is divided into four regions, regions 1 and 3 are compressed horizontally and elongated vertically. This distortion can be understood as a conductor with shorter length and larger cross section; therefore, the conductance is increased. This is equivalent to the situation of increased conductivity with unchanged geometry (Figure 2, middle). Without considering any model geometry variations, the reconstructed image shows regions 1 and 3 as positive conductivity changes (red) (Figure 2 , right). Inversely, regions 2 and 4 are represented as negative conductivity changes (blue). These effects are named "deformation artefacts".

As illustrated in Figure 3: at the $11^{\text {th }}$ frame $(\mathrm{fn}=11$, distortion amplitude is 

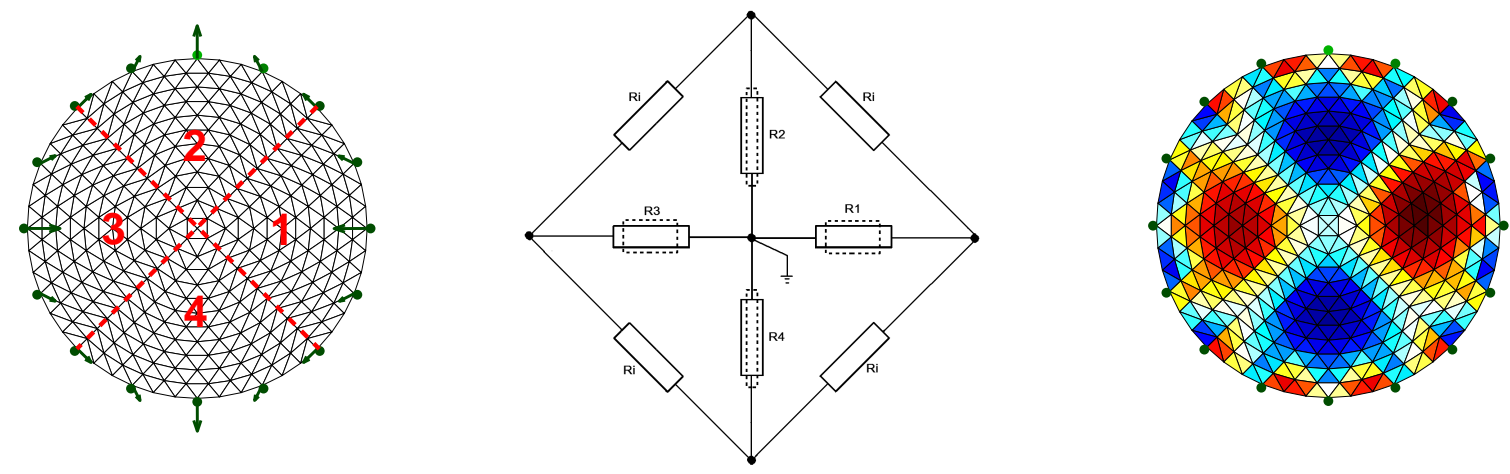

Figure 2. Effect of boundary distortion on EIT reconstruction.

left: a homogeneous medium with a distorted boundary. Four regions are defined to identify different effects.

middle: the simplified circuit model of the medium. $R_{1, \ldots, 4}$ represent resistances of regions $1, \ldots, 4$, respectively. $R_{i}$ are intermediate resistances between two regions. In regions 1 and 3, the boundary distortion can be regarded as shortened and widened resistors (dashed blocks), and in regions 2 and 4 as elongated and thinned resistors. right: image reconstructed using GN method with an assumed static boundary $(\lambda=0.4$, noise-free $)$.

$0.05 \%$ of the model diameter), although temporal solvers show better target resolution, all methods identify target successfully due to the small model deformation. With increasing model deformation ( $\mathrm{fn}=61,0.3 \%$ distortion; $\mathrm{fn}=111,0.55 \%$ distortion), the GN and temporal solvers failed to identify the target due to severe distortion artefacts. The electrode movement solver still shows the target, although artefacts emerge. Further deformation ( $\mathrm{fn}=161,0.8 \%$ distortion) makes the electrode movement solver incapable of recognizing target due to severe artefacts. Compared with other solvers, the temporal and electrode movement solver is much more robust against the "deformation artefacts".

Illustrated in Figure 4, a similar conclusion can be drawn from phantom data reconstructions. With gradually increased deformation $(0 \sim 1.7 \mathrm{~cm}$, approximately from $\mathrm{fn}=1$ to $\mathrm{fn}=75)$, the temporal and electrode movement solver shows the best artefact robustness. The gradual disappearance of the target at the position $(7,0)$ illustrates the "deformation artefacts": the target is gradually obscured by the positive artefacts introduced by boundary deformation.

In order to test the applicability to in vivo measurements, a thirty-second frame sequence was acquired (the frame rate was $13 \mathrm{fps}$, thus 390 frames in total) for a deep-breathing human subject. Data were reconstructed by the temporal and electrode movement solver (Figure 5). The reconstructed image sequence was chosen from the end inspiration to the end expiration. The reference data $\left(\mathbf{v}_{0}\right)$ were chosen as the average of the whole data set. At the end inspiration when $t=17.8 \mathrm{sec}$, the lungs showed conductivity decrease in blue (compared with the reference); during expiration, conductivity gradually increased and, after crossing the reference $\left(\mathbf{x}_{0}\right)$, the lungs regions 


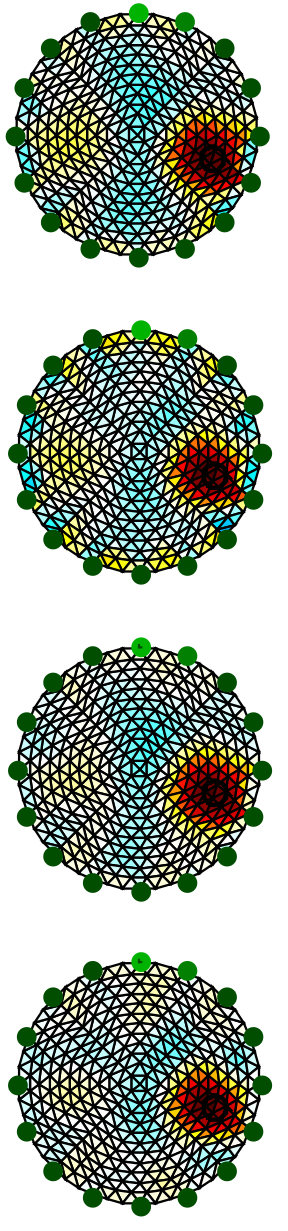

$\mathrm{fn}=11$
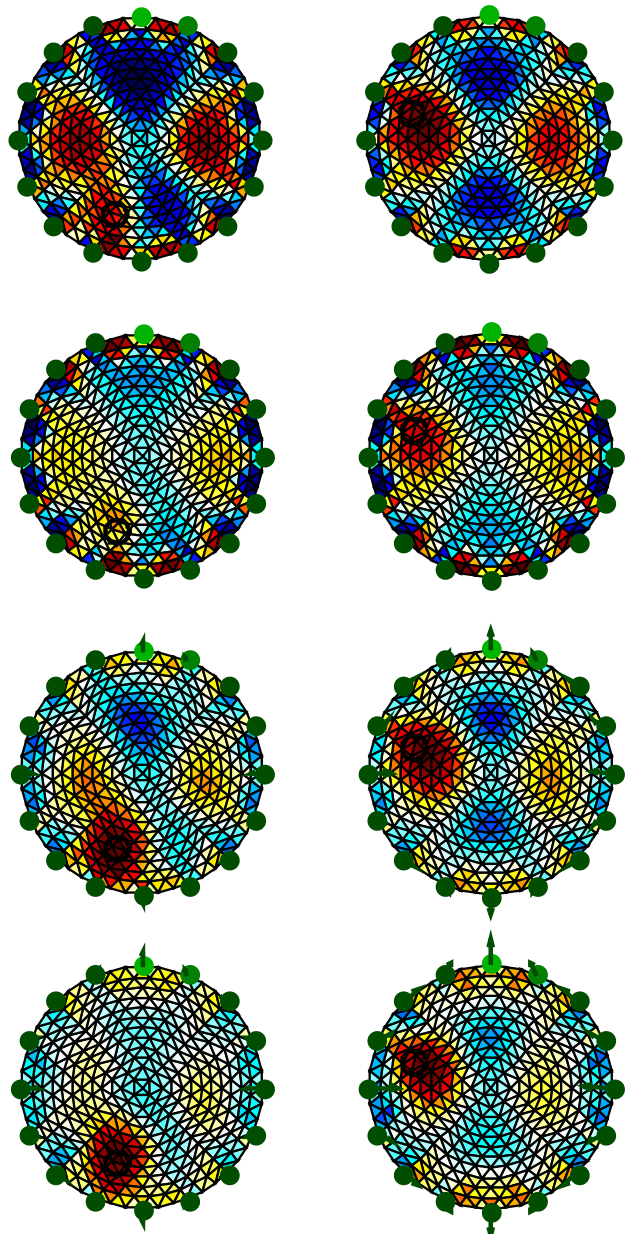

$\mathrm{fn}=61$

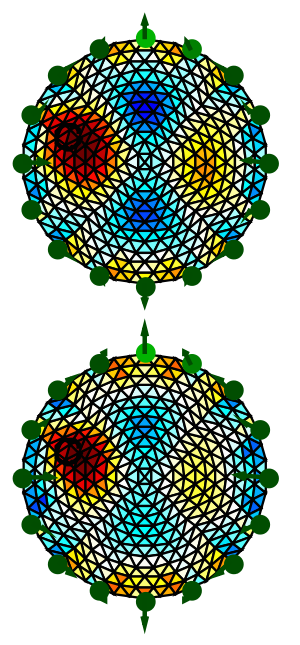

$\mathrm{fn}=111$

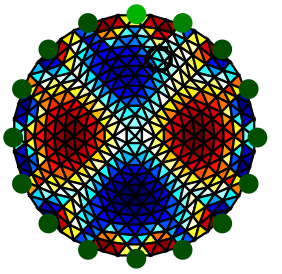

(a)

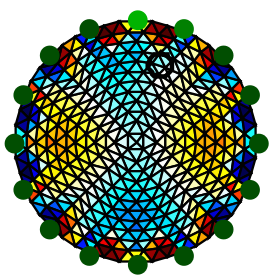

(b)

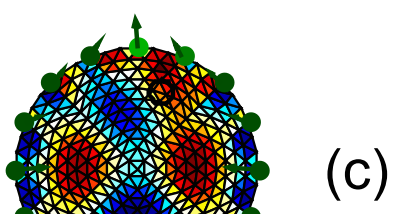

(c)

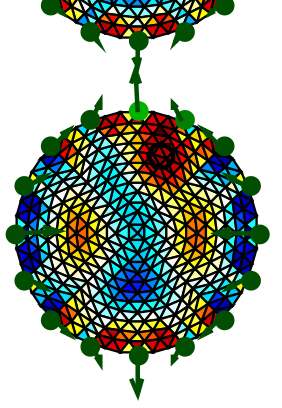

(d)

Figure 3. Images reconstructed from simulated data (using $\lambda=0.4, S N R=10$ ). (a) GN solver; (b) Temporal solver; (c) Electrode movement solver; (d) Temporal and Electrode movement solver. From left to right, the target rotated clockwise (simulated target positions were shown by black circles); the boundary was increasingly distorted and arrows indicate amplitudes and directions of reconstructed electrode movements. Arrow amplitudes are scaled by 20 .

showed increased conductivity (in red); they finally reached the end of expiration at $t=22.8 \mathrm{sec}$ when lungs remained the residue volume and the image showed the highest conductivity.

\section{Discussion}

In this paper, we propose an image processing algorithm to help address two issues in EIT: uncertainty in boundary movement and noise in reconstructed images. Images of the conductivity change and electrode movement are calculated from sequences of 

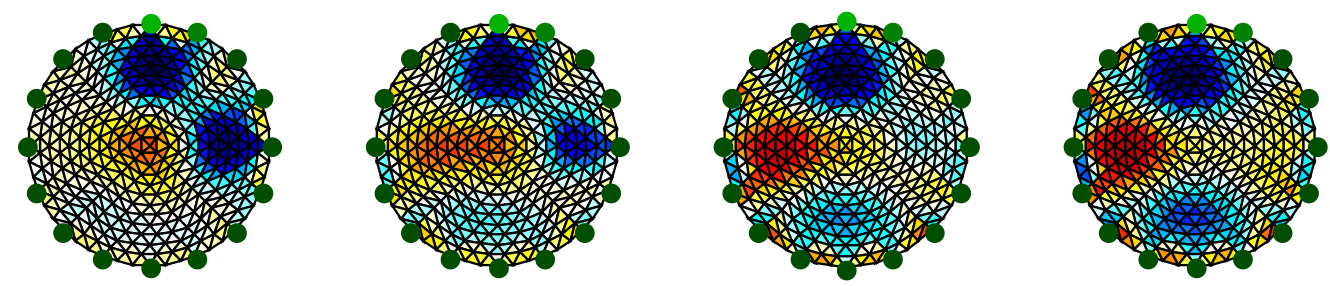

(a)
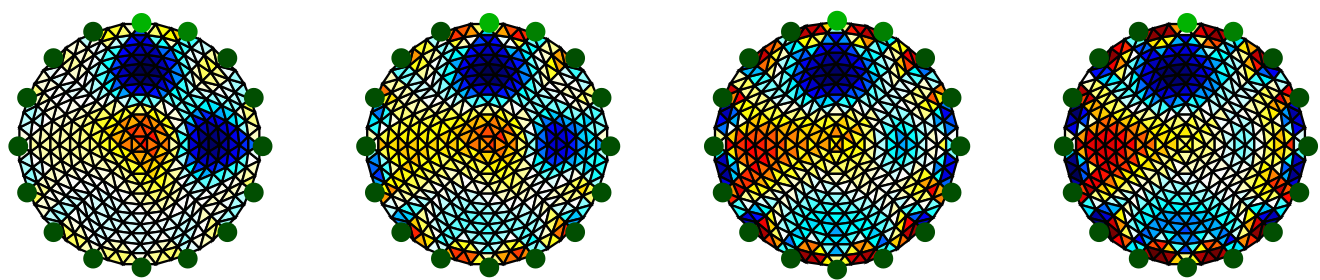

(b)
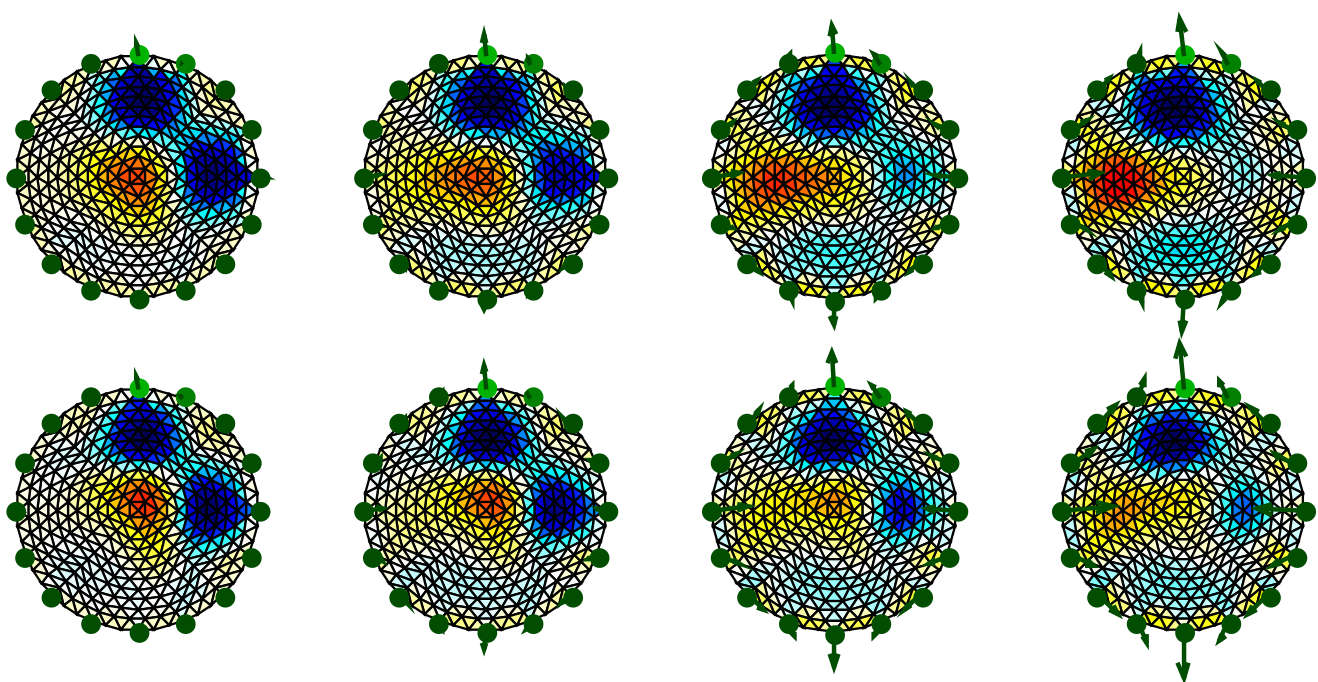

$\mathrm{fn}=31$

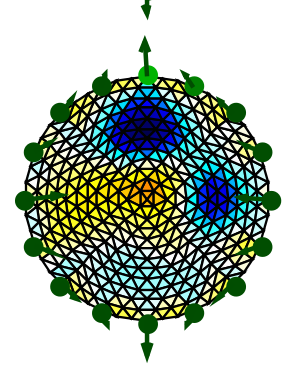

$\mathrm{fn}=41$

$\mathrm{fn}=51$

Figure 4. Images reconstructed from different solvers using measured data from a saline phantom (using $\lambda=0.4$ ). (a) GN solver; (b) Temporal solver; (c) Electrode movement solver; (d) Temporal and Electrode movement solver. From left to right, frames were taken at 21, 31, 41 and 51, corresponding to increasing boundary distortion. Arrows indicate amplitudes and directions of boundary distortion. Arrow amplitudes are scaled by 10 .

EIT data around the current frame. The temporal reconstruction proposed directly formulates both the reconstructed conductivity change and electrode movement in terms of a single regularized inverse, based on a priori models that adjacent images and boundary shapes from a fast measurement system are highly correlated. This method takes advantage of correlations that occur because the conductivity changes and boundary movements happen more slowly than the data acquisition.

In this paper, one important assumption is that the Jacobian is time-invariant. This is a core assumption in time-difference EIT. The model is linearized by taking a derivative at a reference point. Simulations suggest that the changes in the Jacobian 

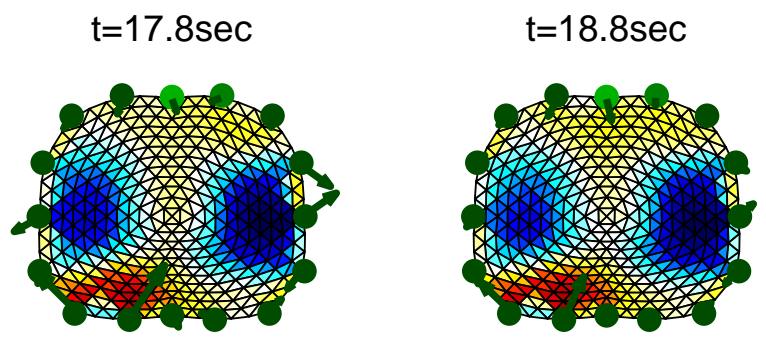

$t=19.8 \mathrm{sec}$
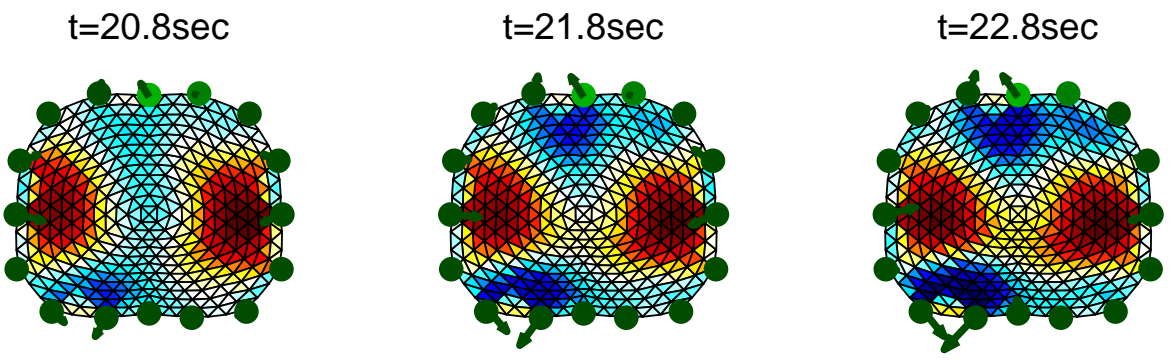

Figure 5. Images of maximal expiration on human subject (using $\lambda=2$ ). Anterior is at image top; left is at image left. Arrows show movement directions and amplitudes that are exaggerated by 30 . The reference signal was calculated as the average over whole data set.

are much less significant than any general inaccuracies in the Jacobian used due to inadequate knowledge of the initial geometry and conductivity distribution.

This result is based on our previous work (Adler et al 2007 and Soleimani et al 2006); the combined solver of temporal inverse and electrode movement reconstruction uses temporal reconstruction method to calculate both the conductivity change and electrode movement from difference EIT data. The novel results demonstrated in this paper are to show significant improvements in noise performance and artefact resistance. The algorithm performed well in simulated and phantom in comparison to reconstructions which consider only the conductivity change, and in comparison to our previous results for electrode movement. Considering the reconstructed images and electrode movement from in vivo human data, this method shows potential to be used in real time monitoring of lung ventilation.

\section{Acknowledgments}

This work was supported by a grant from NSERC Canada.

\section{References}

Adler A, Guardo R and Berthiaume Y 1996 Impedance imaging of lung ventilation: Do we need to account for chest expansion? IEEE Trans. Biomed. Eng. 43(4) 414-20 
Adler A and Guardo R 1996 Electrical impedance tomography: regularized imaging and contrast detection IEEE Trans. Med. Imaging 15 170-179

Adler A and Lionheart W R B 2006 Uses and abuses of EIDORS: An extensible software base for EIT Physiol. Meas. 27 25-42

Adler A, Dai T and Lionheart W R B 2007 Temporal image reconstruction in electrical impedance tomography Physiol. Meas. 28 S1-S11

Barber D C and Brown B H 1988 Errors in reconstruction of resistivity images using a linear reconstruction technique Clin. Phys. Physiol. Meas. 9(suppl. A) 101-4

Campbell J H, Harris N D, Zhang F, Brown B H and Morice A H 1994 Clinical applications of electrical impedance tomography in the monitoring of changes in intrathoracic fluid volumes Physiol. Meas. 15 A $217-\mathrm{A} 22$

Cheney M, Isaacson D, Newell J C, Simske S and Goble J C 1990 NOSER: an algorithm for solving the inverse conductivity problem Int.J.Imaging Syst.Technol. 2 66-75

Dai T, Soleimani M and Adler A 2007 Four-Dimensional Regularization for Electrical Impedance Tomography Imaging (IFMBE Proceedings, vol 17, VIII Conference on Electrical Impedance Tomography, Aug 29-Sep 2, 2007, Graz, Austria, p 408

Dijkstra A M, Brown B H, Leathard A D, Harris N D, Barber D C and Edbrooke D L 1993 Clinical applications of electrical impedance tomography J. Med. Eng. Technol. 17(3) 89-98

Eyuboglu B M, Brown B H and Barber D C 1989 In vivo imaging of cardiac related impedance changes IEEE Eng. Med. Biol. Mag. 8(1) 39-45

Fahrmeir L, Tutz G 1994 Multivariate Statistical Modeling Based on Generalized Linear Models. New York: Springer-Verlag.

Gómez-Laberge C and Adler A 2007 Direct calculation of the electrode movement Jacobian for 3D EIT VIII Conf Electrical Impedance Tomography, Graz, Austria, Aug 29-Sept 2, 364-7

Graham B and Adler A 2007 Electrode Placement Configurations for 3D EIT Physiol. Meas. 28 S29-S44

Harris N D, Suggett A J, Barber D C and Brown B H 1988 Applied potential tomography: a new technique for monitoring pulmonary function Clin. Phys. Physiol. Meas. 9 79-85

Holder D S 1987 Feasibility of developing a method of imaging neuronal activity in the human brain: a theoretical review Med. Biol. Eng comput. 25 2-11

Lionheart W, Polydorides N and Borsic A 2005 Why is EIT so hard, in Electrical impedance tomography: methods, history and applications, Holder D S, Ed. Bristol and Philadelphia, IOP, pp. 3-4

McArdle F J, Suggett A J, Brown B H, and Barber D C 1988 An assessment of dynamic images by applied potential tomography for monitoring pulmonary perfusion Clin. Phys. Physiol. Meas. 9, 87-91

Olsen P A and Gopinath A R 2004 Modeling inverse covariance matrices by basis expansion IEEE Trans Speech Audio Processing 12 37-46

Polydorides N and Lionheart W R B 2002 A Matlab toolkit for three-dimensional electrical impedance tomography: A contribution to the Electrical Impedance and Diffuse Optical Reconstruction Software project Meas. Sci. Technol. 13 1871-83

Soleimani M, Gomez-Laberge C and Adler A 2006 Imaging of conductivity changes and electrode movement in EIT Physiol. Meas. 27 S103-S13

Tidswell A T, Gibson A, Bayford R H and Holder D S 2001 Electrical impedance tomography of human brain activity with a two-dimensional ring of scalp electrodes Physiol. Meas. 22 167-75

Vauhkonen M, Vadàsz D, Karjalainen P A, Somersalo E and Kaipio J P 1998a Tikhonov regularization and prior information in electrical impedance tomography IEEE Trans Med Imaging 17 285-93

Vauhkonen M, Karjalainen P A and Kaipio J P 1998b A Kalman filter approach to track fast impedance changes in electrical impedance tomography IEEE Trans. Biomed. Eng $\mathbf{4 5}$ 486-93

Vonk-Noordegraaf A, Janse A, Marcus J T, Bronzwaer J G, Postmus P E, Faes T J C and de Vries P M 2000 Determination of stroke volume by means of electrical impedance tomography Physiol. Meas. 21 285-93

Wilkinson A J, Randall E W, Cilliers J J, Durrett D R, Naidoo T and Long T 2005 A 1000-measurement 
frames/second ERT data capture system with real-time visualization, IEEE Sensors Journal 5(2) 300-7

Yorkey T J, Webster J G and Tompkins W J 1987 Comparing reconstruction algorithms for electrical impedance tomography IEEE Trans. Biomed. Eng 34 843-52

Zhang J and Patterson R P 2005 EIT images of ventilation: what contributes to the resistivity changes? Physiol. Meas. 26 S81-S92 